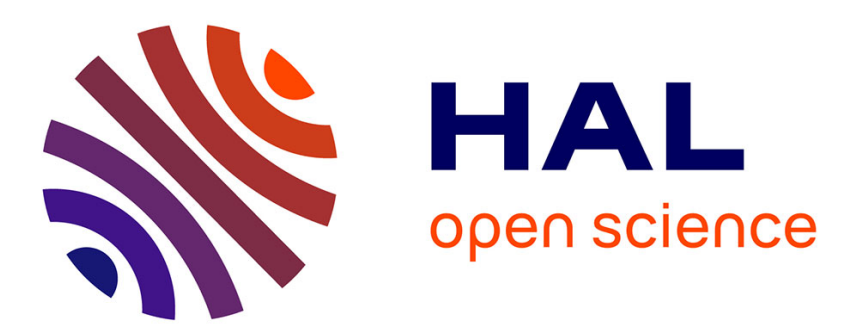

\title{
Exploiting bistable oscillator subharmonics for magnified broadband vibration energy harvesting
}

Thomas Huguet, Adrien Badel, Mickaël Null Lallart

\section{To cite this version:}

Thomas Huguet, Adrien Badel, Mickaël Null Lallart. Exploiting bistable oscillator subharmonics for magnified broadband vibration energy harvesting. Applied Physics Letters, 2017, 111, pp.173905. 10.1063/1.5001267 . hal-01764325

\section{HAL Id: hal-01764325 \\ https://hal.science/hal-01764325}

Submitted on 31 Oct 2018

HAL is a multi-disciplinary open access archive for the deposit and dissemination of scientific research documents, whether they are published or not. The documents may come from teaching and research institutions in France or abroad, or from public or private research centers.
L'archive ouverte pluridisciplinaire HAL, est destinée au dépôt et à la diffusion de documents scientifiques de niveau recherche, publiés ou non, émanant des établissements d'enseignement et de recherche français ou étrangers, des laboratoires publics ou privés. 


\title{
Exploiting bistable oscillator subharmonics for magnified broadband vibration energy harvesting
}

\author{
Thomas Huguet, ${ }^{1,2}$ Adrien Badel, ${ }^{2, a)}$ and Mickaël Lallart ${ }^{1}$ \\ ${ }^{1}$ Univ. Lyon, INSA-Lyon, LGEF, EA682, F-69621, VILLEURBANNE, FRANCE \\ ${ }^{2}$ Univ. Savoie Mont-Blanc, SYMME, F-74000, ANNECY, FRANCE
}

\begin{abstract}
Recent research on primary battery alternatives for supplying autonomous wireless devices have recently highlighted the advantages of nonlinear oscillators' dynamics and more particularly bistable oscillators' behavior for ambient vibration harvesting. The key property of bistable oscillators compared to linear ones is their enhanced operational frequency bandwidth under harmonic excitation, potentially leading to a better adaptation to the environment. However, the classical frequency response characterization of such devices does not reveal all the possible dynamic behaviors offered by bistable oscillators. Thus, subharmonic motions are experimentally investigated in this letter, and their energy harvesting potentials as well as their ability to enhance the bistable generator bandwidth are evaluated. Results obtained with a generator integrating buckled beams for the bistability feature show that, in addition to the commonly considered harmonic behavior, subharmonics allow widening the bistable microgenerator useful operating frequency band by $180 \%$ compared to the sole exploitation of the first harmonic motion.
\end{abstract}

As the spreading of stand-alone, left-behind devices is facing the challenge of energy supply, vibration energy harvesting is an attractive alternative to batteries, hence improving their compactness (no need to embed the whole lifetime energy), autonomy (no need to replace depleted batteries) and adaptability to harsh conditions such as high temperature environments where electrochemical batteries are not suitable. Because of its good trade-off between simplicity and efficiency, a particularly appreciated solution is to scavenge ambient vibrational energy by coupling a seismic oscillator to the vibrating source and transfer a part of the ambient mechanical energy to the inertial mass of the oscillator. Then, an electromechanical transducer (e.g., piezoelectric material or coil-magnet interaction) is used to convert the mechanical energy within the oscillator into electricity. More particularly, linear oscillators are used to amplify ambient vibration and show optimal performance when the ambient vibration spectrum matches their resonant frequency ${ }^{1}$. However, the amplification effect dramatically collapses when moving away from the resonance peak, which is a drastic drawback when the operating frequency is not perfectly constant or under random excitation.

To overcome this drawback, a significant attention has been recently placed on nonlinear bistable oscillators for vibration energy harvesting purpose $^{2,3}$. The main asset offered by bistability compared to linear systems is that, for a sinusoidal vibration source and for an adequate ambient acceleration magnitude, bistable oscillators show a wider frequency bandwidth for a similar maximum harvested power ${ }^{4}$. These microgenerators are usually characterized by their hysteretic frequency response obtained by slowly sweeping the excitation frequency up and down and recording the harvested power as a function of the frequency.

However, this characterization method may not be representative of realistic excitation and does not reveal all the possible behaviors of bistable

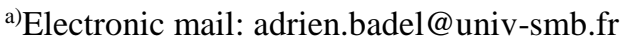



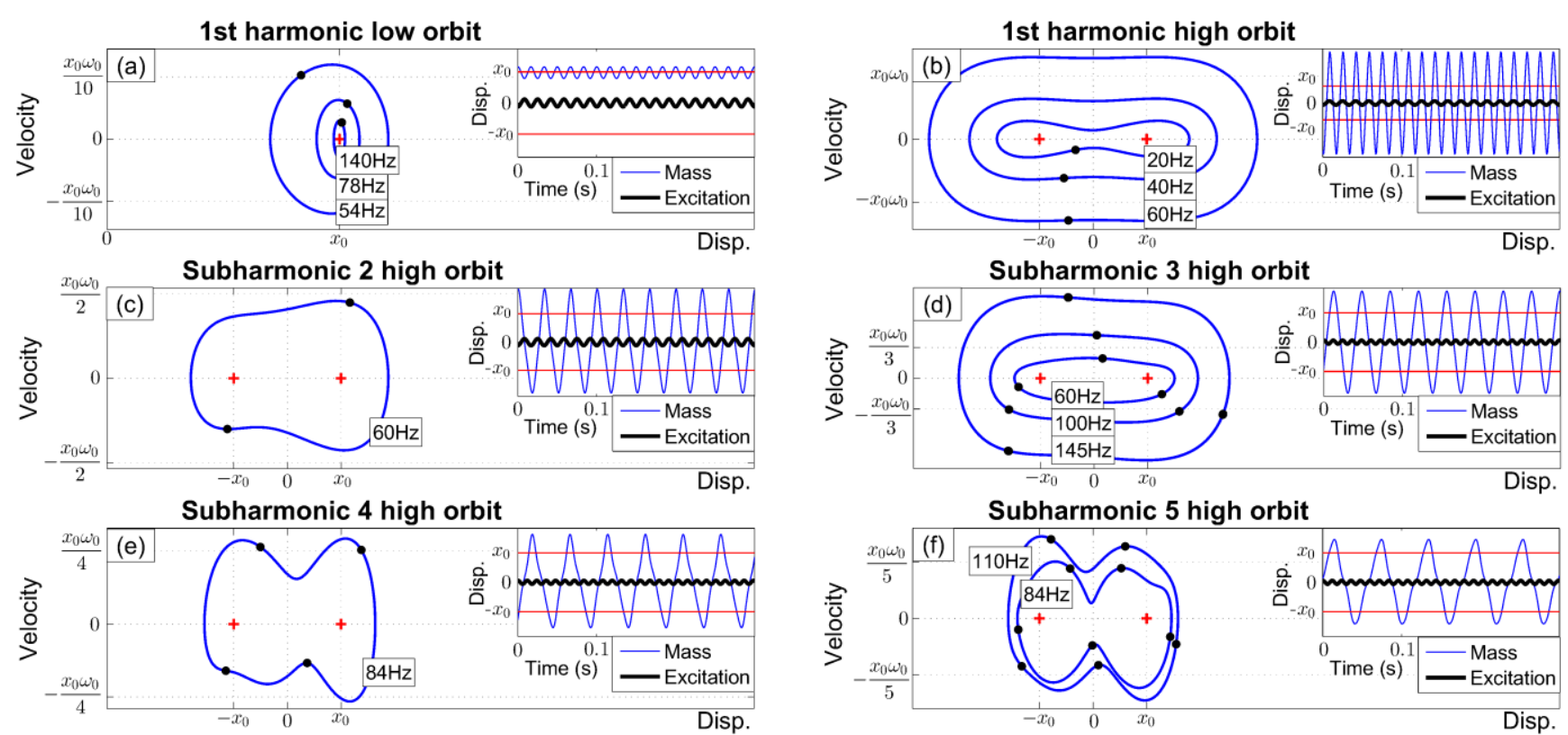

Figure 1: Numerical phase portraits of different bistable oscillator orbits with their respective Poincaré section (black dots) and their respective time signal waveform.

oscillators. In particular, subharmonic behaviors have been briefly pointed out in purely mechanical studies $^{5,6,7,8}$, but were mainly regarded as curiosities without applicative analyses. Nevertheless, those special bistable oscillator behaviors happen on certain frequency ranges, which could widen the global mechanical bandwidth. Hence, A. Arietta et al. ${ }^{9}$ and A. Syta et al. ${ }^{10}$ studied the energy harvested on one of these subharmonic motions at a particular frequency but does not evaluate its full frequency range.

In this letter, we propose to go further by experimentally focusing on several bistable oscillator subharmonic motions for energy harvesting purposes and to evaluate their full frequency ranges to appreciate their contribution on the global generator bandwidth.

W.-Y. Tseng and al. ${ }^{11}$ showed in 1971 that bistable oscillator's behaviors can be described by a Duffing-type mechanical equation ${ }^{12}$ as follows:

$$
\ddot{x}+\frac{1}{2} \omega_{0}^{2}\left(\frac{x^{2}}{x_{0}^{2}}-1\right) x+\frac{\omega_{0}}{Q} \dot{x}=-\gamma \cos (\omega t)
$$

with $x$ the mass relative position with respect to the support, $x_{0}$ the mass relative first stable position (the second being $-x_{0}$ ), $\omega_{0}$ the natural angular frequency and $Q$ the mechanical quality factor. $\gamma$ and $\omega$ are the ambient acceleration and the ambient angular frequency, respectively. Contrary to linear oscillators, for a given ambient excitation, a nonlinear oscillator can have different steady-state behaviors referred as attractors or orbits. These are reached depending on the nonlinear oscillator initial conditions (mass initial position and velocity). A numerical resolution of Equation (1) is conducted to illustrate these different orbits. Figure 1 depicts the time-domain waveforms showing the mass motion as well as the phase portraits and their respective Poincaré section (black dots) for each behavior reached with a given set of initial conditions and for different ambient frequencies. The parameters used for these simulations correspond to the prototype presented in the experimental part and are listed in Table I.

Table I: Simulation parameter values.

\begin{tabular}{lccl}
\hline \multicolumn{1}{c}{ Parameter } & Symbol & Value & Unit \\
\hline Mass stable positions & $\pm x_{0}$ & \pm 290 & $\mu \mathrm{m}$ \\
Natural angular frequency & $\omega_{0}$ & 230 & $\mathrm{rad} / \mathrm{s}$ \\
Mechanical quality factor & $Q$ & 60 & - \\
Acceleration magnitude & $\gamma$ & 5 & $\mathrm{~m} / \mathrm{s}^{2}$ \\
\hline
\end{tabular}

In Figures 1(a) and 1(b), the mass moves at the same frequency as the excitation; such behavior 
correspond to the classical $1^{\text {st }}$ harmonic orbit motion. In Figure 1(a), the mass stays around one stable position, leading to low orbit (intra-well) motion. On the opposite, in Figure 1(b), the mass moves around the two stable positions, yielding high orbit (inter-well) motion. The $1^{\text {st }}$ harmonic low and high orbits are the commonly studied orbits, exhibited when using the frequency sweep characterization method. However, at certain frequencies, changing the mass initial conditions leads to behaviors illustrated in Figures 1(c) to 1(f), which are not revealed by the frequency sweep method. For those orbits, the mass moves with a frequency divided by an integer value compared to the excitation. Those behaviors correspond to subharmonic orbits (subharmonic 2 to subharmonic 5 in Figures $\mathbf{1}(\mathrm{c})$ to $\mathbf{1}(\mathbf{f})$ ). In these examples, only subharmonic high orbits are shown (even if subharmonics low orbits also exist), as high orbits are more interesting for energy harvesting because they induce higher mechanical energy. For such a reason, low orbits are not investigated in this letter, with the exception of the $1^{\text {st }}$ harmonic low orbit for the following two reasons: (1) subharmonic low orbit frequency ranges are generally included in the $1^{\text {st }}$ harmonic one so the later represents the whole frequency range on which a low orbit behavior exists; (2) it gives a good reference to appreciate the energy harvested from high orbits.

All the bistable oscillator orbits require appropriate initial conditions to appear. For example, changing the generator initial conditions when the oscillator is moving can be obtained by applying a sharp disturbance on the mass motion, which can thus lead to an orbit hopping phenomenon. Such a disturbance is required as subharmonic behaviors all coexist with the $I^{\text {st }}$ harmonic orbit, so that they might not be revealed by slow frequency sweep characterizations commonly used in the literature as stated before. On the opposite, $1^{\text {st }}$ harmonic low and high orbits exist alone on certain frequency ranges and naturally appear with this characterization method.

The bistable generator prototype, presented in Figure 2(a), is made of two parallel steel beams clamped on both sides. A brass proof mass with two magnets is attached at the center of the beams.
Bistability is obtained through buckling the beams thanks to a micrometer screw placed at one end. Prototype dimensions are detailed in Table II. It can be noted that the small value of the buckling coefficient $(\sim 1 / 100)$, yielding a mass displacement amplitude that stays small compared to the length of beams during experiments, ensures the validity of the Duffing-type mechanical equation for correctly describing the system.

Table II: Experimental parameter values.

\begin{tabular}{lcl}
\hline \multicolumn{1}{c}{ Parameter } & Value & Unit \\
\hline Inertial mass (magnets included) & 30 & $\mathrm{~g}$ \\
Brass mass dimensions & $30 \times 10 \times 10$ & $\mathrm{~mm}^{3}$ \\
Beams dimensions & $28 \times 10 \times 0.10$ & $\mathrm{~mm}^{3}$ \\
Buckling distance reduction & 1.5 & $\mu \mathrm{m}$ \\
Mass stable position & \pm 290 & $\mu \mathrm{m}$ \\
\hline
\end{tabular}

The global setup, shown in Figure 2(b), is composed of an electrodynamic shaker for excitation, a differential laser vibrometer, an accelerometer and a coil. The seismic shaker is driven by a real-time controller (DSPace) to insure a constant amplitude sinusoidal acceleration thanks to a feedback loop. The relative displacement of the mass with respect to the shaker is measured with the differential laser vibrometer. The coil has two functions: (1) applying a pulse force (through a pulse voltage) on the magnets, which allows changing the initial conditions of the bistable oscillator and therefore possibly observing subharmonic behavior; (2) harvesting energy by connecting a resistance, as it allows mechanical energy conversion into electrical energy through magnetic interaction between the coil and the magnet. Noting that, in the operating frequency range, the output impedance of the generator is almost purely resistive, the added resistance has been set to match the internal resistance of the coil $(18 \Omega)$ to optimize the energy transfer from the coil to the load. It can be noted that although a pure resistance may not represent a full energy harvesting electrical interface, it allows a good estimation of the harvestable energy.

The experimental process to reach and characterize the bistable generator orbits consists in the following steps: (1) set an excitation amplitude and frequency; (2) apply rectangular $5 \mathrm{~ms}$ voltage pulses of different amplitudes (10 to 
$50 \mathrm{~V}$ ) at various phases on the coil to investigate the different behaviors at this frequency (the sharp disturbance applied through the pulse allows orbit changes contrary to smooth sweep up and down frequency characterization); (3) when an orbit of interest is reached, change slowly and smoothly the excitation frequency (up or down) to a new one. If the frequency change is smooth enough, the system stays on the same orbit if it exists at this new frequency. If the steady-state behavior changes, then it can be assumed that the previous steadystate behavior does not exist at this frequency. Using this method, the frequency ranges for all the studied generator behaviors can be determined; (4) when the system is in steady state motion, the average harvested power $P_{\text {mean }}$ can be evaluated through the root mean square value of the measured voltage $V_{R M S}$ over the load resistance $r$ with the following relation: $P_{\text {mean }}=V_{r m s}^{2} / r$.

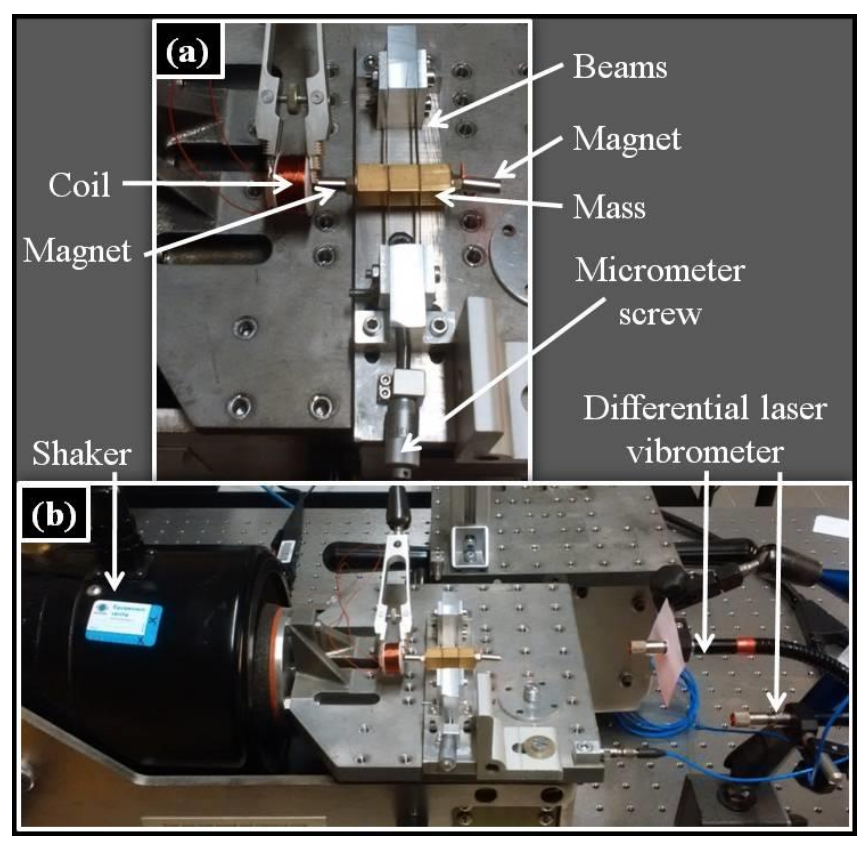

Figure 2: (a) Bistable generator prototype and (b) experimental setup.

The results obtained with the proposed approach for a $5 \mathrm{~m} / \mathrm{s}^{2}$ sinusoidal base acceleration amplitude are detailed in Figure 3, depicting the $1^{\text {st }}$ harmonic low and high orbits, the $3^{\text {rd }}$ subharmonic high orbit and the $5^{\text {th }}$ subharmonic high orbit. Subharmonics 2 and 4 are not presented, as they appear on a narrow bandwidth with significantly lower magnitude than other existing orbits. Hence, they do not represent good candidates for energy harvesting. Subharmonic low orbits are also not presented because of their low associated energy. Figure 3(a) presents the mass displacement amplitude for each orbit as a function of the excitation frequency and Figure 3(b) presents the harvested power dissipated in the $18 \Omega$ resistance for each orbits as a function of the excitation frequency. Finally, an example of the experimental displacement waveforms, phase spaces and Poincaré sections is shown in Figure 4 for each considered bistable oscillator behavior. Considering the process to reach the different behaviors, the repeatability of the orbit jumps and the consequent time spent on each orbit for measurements, all the orbits presented here are considered as relatively robustly stable.
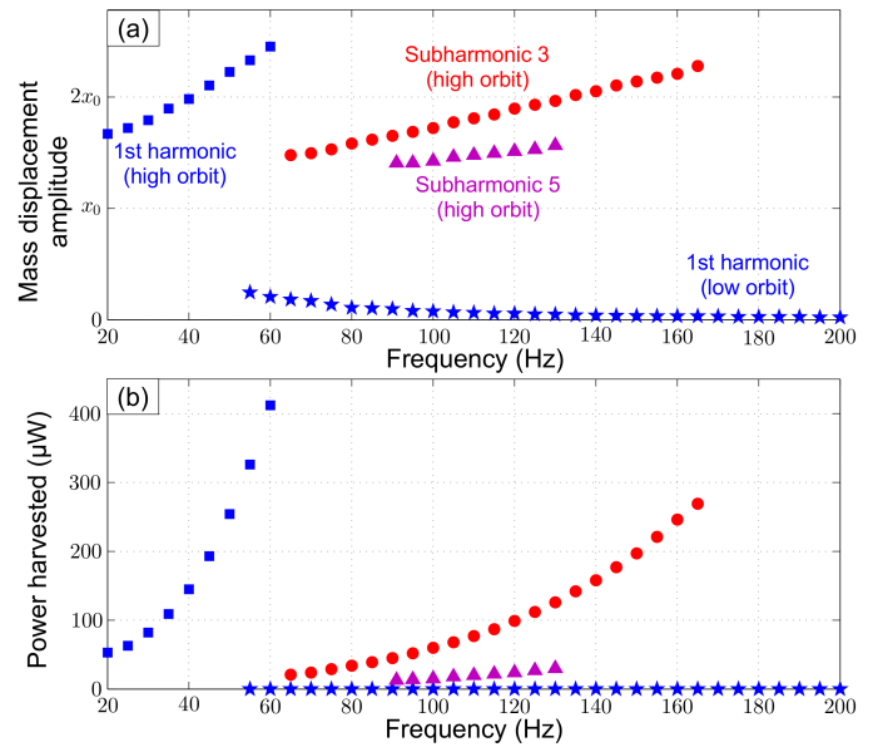

Figure 3: Experimental excitation frequency dependence of (a) the generator mass displacement amplitude and (b) the power harvested through the $18 \Omega$ resistance.

Obtained results clearly demonstrate the advantage of using subharmonic 3 high orbit (Figure 3(b)), where the maximal power harvested $(269 \mu \mathrm{W})$ corresponds to $65 \%$ of the $1^{\text {st }}$ harmonic maximal harvested power $(412 \mu \mathrm{W})$ on a frequency range where the $1^{\text {st }}$ harmonic behavior only features low orbit motion. Subharmonic 5 high orbit does not present any interest, as it exists in a narrower range included within the $3^{\text {rd }}$ subharmonic and exhibits lower performance (30 $\mu \mathrm{W}$ maximum harvested power). It is interesting to 
note that the frequency ranges of the predominant high orbits, namely the $1^{\text {st }}$ harmonic and the subharmonic 3 orbits, appears in the neighborhood of the natural frequency $(37 \mathrm{~Hz})$ and 3 times the natural frequency $(111 \mathrm{~Hz})$.

As the main benefit of using nonlinear oscillators is to enhance the operating frequency range of the generator compared to linear oscillators, this study suggests that such advantage can be further magnified through the use of subharmonic 3 that appears to have an interesting contribution to this asset: the bistable experimental device harvests more than $100 \mu \mathrm{W}$ over a $70 \mathrm{~Hz}$ frequency range considering both ${ }^{\text {st }}$ harmonic and $3^{\text {rd }}$ subharmonic high orbits (from $35 \mathrm{~Hz}$ to $60 \mathrm{~Hz}$ and from $120 \mathrm{~Hz}$ to $165 \mathrm{~Hz}$ ) instead of $25 \mathrm{~Hz}$ for the sole $1^{\text {st }}$ harmonic, leading to a $180 \%$ increase.
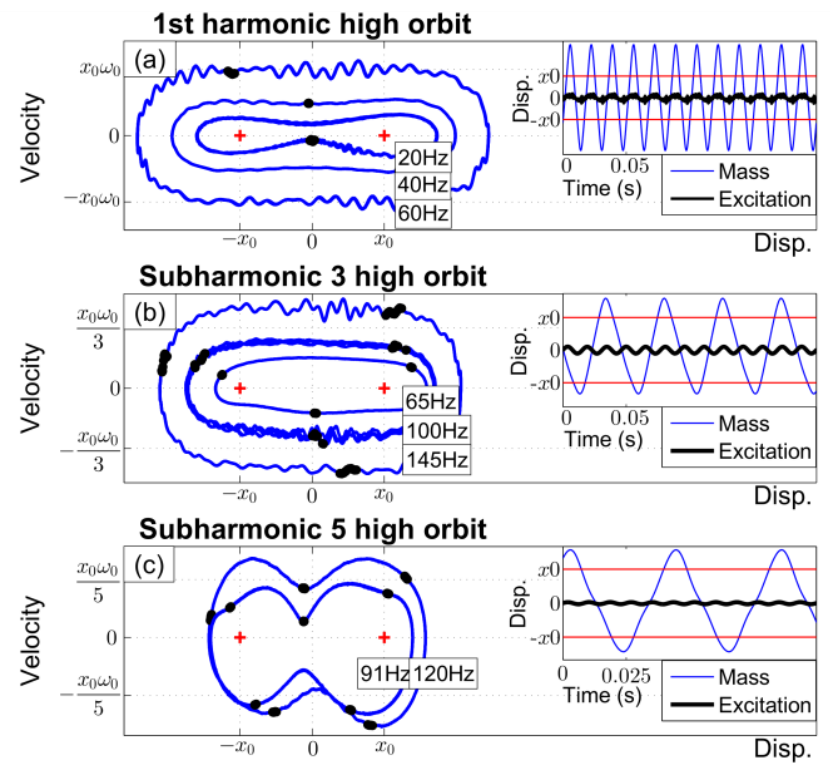

Figure 4: Experimental phase portraits with their respective Poincaré section (black dots) and their respective time signal waveform.

In conclusion, this Letter demonstrated that exploiting subharmonic behaviors of bistable oscillators in the framework of mechanical energy harvesting would allow a widened useful operating frequency band, therefore allowing the generator to adapt in a better way to his environment, hence being able to supply microelectronic devices such as sensor networks with more efficiency. Based on these experimental results, future works will focus on the theoretical investigation of these subharmonic motions to evaluate and optimize the bandwidth enhancement as well as to discuss their robust stability through, for instance, a basin of attraction analysis.

The authors acknowledge the support of Région Rhône-Alpes through the ARC 4 Energies program.

${ }^{1}$ C.B. Williams \& R.B. Yates, Sensor and Actuators A: Physical 52, 8 (1996)

${ }^{2}$ J. Baker, S. Roundy \& P. Wright, 3rd International Energy Conversion Engineering Conference (San Francisco, California 2005)

${ }^{3}$ A. Erturk, J. Hoffmann \& D.J. Inman, Applied Physics Letters 94, 254102 (2009)

${ }^{4}$ R.L. Harne \& K.W. Wang, Smart Materials and Structures 22, 023001 (2013)

${ }^{5}$ F.C. Moon \& S.W. Shaw, Non-Linear Mechanics 18, 465 (1983)

${ }^{6}$ S.A. Emam \& A.H. Nayfeh, Nonlinear Dynamics 35, 105 (2004)

${ }^{7}$ A.F. Arrieta, S.A. Neild \& D.J. Wagg, Nonlinear Dynamics 58, 259 (2009)

${ }^{8}$ S.N. Mahmoodi, N. Jalili \& M. Ahmadian, Nonlinear Dynamics 59, 397 (2010)

${ }^{9}$ A.F. Arrieta, P. Hagedorn, A. Erturk \& D.J. Inman, Applied Physics Letters 97, 104102 (2010)

${ }^{10}$ A. Syta, G. Litak, M.I. Friswell \& S. Adhikari, The European Physical Journal B 89, 99 (2016)

${ }^{11}$ W.-Y. Tseng \& J. Dugundji, Journal of Applied Mechanics 38, 467 (1971)

${ }^{12} \mathrm{G}$. Duffing, Zeitschrift für Angewandte Mathematik und Mechanik 1, 72 (1921) 